\title{
Eine Verfassung für Laos
}

Von Helmut Arndt

\section{Vorbemerkung}

Laos, im Norden Indochinas zwischen Thailand und Vietnam gelegen, ein schwer zugängliches Binnenland, flächenmäßig fast so groß wie die Bundesrepublik, jedoch nur von rund vier Millionen Menschen bewohnt, befindet sich seit 1975 nach der Abschaffung der Monarchie im verfassungslosen Zustand. Ein jetzt vorgelegter Entwurf der Obersten Volksversammlung, der zum Jahresende in Kraft gesetzt werden soll, bedeutet eine neue Etappe in der wechselvollen Geschichte des einstigen Reichs der Millionen Elefanten.

1353 gilt als das Gründungsdatum des Königreichs Lane Xang, das in seiner Blütezeit von Yünnan bis Kambodscha, von Nordthailand bis Annam reichte. Zu Beginn des 18. Jahrhunderts zerfiel Laos in die Teilkönigreiche von Luang Prabang, Vientiane und Champassak, die nach wechselnder birmanischer und annamitischer Oberhoheit zu Beginn des 19. Jahrhunderts unter siamesische Souveränität gerieten und 1893 französisches Protektorat wurden.

Die staats- und völkerrechtlichen Verhältnisse Laos' erfuhren bis 1933 in einer Reihe von (ungleichen) Verträgen mit der Protektoratsmacht ihre Regelung, aufgrund derer Laos Teil von Französisch-Indochina wurde.

1941 wurde ein neuer Protektoratsvertrag zwischen dem König von Laos und der Regierung in Vichy geschlossen, doch der japanische Einfall in Indochina im gleichen Jahr und die Annektion laotischer Provinzen durch Siam führten zur Bildung einer nationalen Bewegung, die die Bande zu Frankreich lockerte und 1945 die Unabhängigkeit proklamierte. Nach der Kapitulation Japans 1945 und der Rückkehr der Franzosen kam es zum Abschluß eines modus vivendi, der durch ein französisch-laotisches Abkommen vom 27. August 1946 institutionalisiert wurde. Eine verfassunggebende Nationalversammlung wurde gewählt, die die Verfassung der Monarchie ausarbeitete und am 11.5.1947 verabschiedete; diese blieb bis 1975 - mit den Änderungen von 1956 - in Kraft.

1949 wurde Laos als quasi unabhängiger Staat Mitglied der Französischen Union. 1954 erlangte es zugleich mit Auflösung der Zollunion mit Vietnam und Kambodscha vertraglich von Frankreich die volle Unabhängigkeit, die durch seine Neutralisierung aufgrund der 1. 
Indochina-Konferenz von Genf 1954, die den französisch-vietnamesischen Krieg beendete, in der Erklärung vom 21.7.1954 seine Bestätigung fand.

Das Land lebte in einem schwelenden Bürgerkrieg, in dem sich die königlichen Truppen und die von Nordvietnam - später auch der VR China - unterstützten Pathet-Lao-Streitkräfte dess Prinzen Souphanouvong bekämpften und der durch äußere Einwirkung allmählich der Kontrolle der Laoten entglitt. Am 23.7.1962 kam es in Genf zu einer erneuten Neutralitätserklärung der königlich laotischen Regierung und einem Protokoll der übrigen 13 an der Konferenz teilnehmenden Mächte zur laotischen Neutralität. Dennoch wurde Laos in zunehmendem Maße zum Nebenkriegsschauplatz des amerikanischen Krieges in Vietnam.

Mit Unterstützung starker nordvietnamesischer Verbände erlangte der Pathet Lao schließlich die Kontrolle über drei Fünftel des Staatsgebietes, überwiegend Bergland im Norden und Osten, und über ein Drittel der Gesamtbevölkenung.

1973 wurden die Kampfhandlungen in Laos eingestellt und eine Koalitionsregierung der drei am Krieg beteiligten Parteien gebildet - der dritte Versuch einer Aussöhnung nach den aus den Genfer Konferenzen von 1954 und 1962 hevorgegangenen Regierungen. Die nordvietnamesischen Truppen blieben jedoch im Land. Nach dem Fall von Phnom Penh und Saigon unternahm der Pathet Lao eine militärische Offensive, die 1975 zur faktischen Úbernahme der Regierungsgewalt der Vientiane führte. Der Pathet Lao behielt die Koalitionsregierung unter Prinz Souvanna Phouma zunächst der Form bei, übernahm aber nach und nach Verwaltung und Polizéi. Viele Laoten, besonders der Ober- und Mittelschicht, verließen das Land.

Am 2. Dezember 1975 beschloß ein revolutionärer "Nationalkongreß", die Monarchie abzuschaffen und eine Volksrepublik auszurufen. König Savang Vatthana dankte ab. Kaysone Phomvihane, der Generalsekretär der "Revolutionären Volkspartei", übernahm das Amt des Ministerpräsidenten. Eine Oberste Volksversammlung unter Vorsitz von Prinz Souphanouvong wurde eingesetz und beauftragt, eine Verfassung auszuarbeiten.

\section{Der Verfassungsentwurf - Allgemeines}

Der Entwurf einer Verfassung für die Demokratische Volksrepublik Laos (DVL) wurde am 15. Mai 1990 erstmals publik gemacht, nachdem er von der Verfassungskommission der Obersten Volksversammlung (OVV) ausgearbeitet und verabschiedet worden war.

Der Verfassungsentwurf (VE) beruht nach langjährigen Vorarbeiten, die bis in die Zeit nach dem Sturz der Monarchie zurückgehen (1975), und in deren Verlauf auch Juristen aus 
der DDR bereits einen ersten Entwurf vorgelegt hatten, primär auf Verfassungsmustern der Sow jetunion, Vietnams und der Volksdemokratien des ehemals "sozialistischen Lagers".

Der VE soll dem nun bald 15-jährigen verfassungslosen Zustand der DVL ein Ende bereiten, nachdem die monarchische Verfassung des Königreiches Laos vom 11.5.1947 mit ihren zahlreichen Änderungen durch den Umsturz vom 2.12.1975 außer Kraft gesetzt worden war. Daß man in der Verfassungskommission mit Hochdruck an der Verabschiedung des VE gearbeitet hat, mag auch an den infolge der Politik der Offenen Tür sich steigernden Forderungen nach Rechtssicherheit im Zusammenhang stehen; für ausländische Investoren hing das laotische Investitionsgesetz vom Juli 1988 ohne verfassungsrechtlichen und judiziellen Rahmen quasi in der Luft, den Bestimmungen des Gesetzes fehlte jegliche konstitutionell gesicherte Sanktion.

Der VE liegt nunmehr den gesellschaftlichen Institutionen, den Selbstverwaltungskörperschaften und den Massenorganisationen, damit theoretisch der gesamten Bevölkerung, zur Billigung vor. Nach Abschluß dieses plebiszitären Prozesses Ende August 1990 wird der VE in die OVV eingebracht und soll nach parlamentarischer Beratung hier so rechtzeitig verabschiedet werden, daß die Verfassung am 2.12.1990 zum 15. Jahrestag des Bestehens der DVL in Kraft treten kann.

Parallel dazu gehen die Beratungen über ein neues Zivilgesetzbuch sowie die Ausarbeitung eines neuen Arbeits- und Sozialrechts weiter, nachdem es am 23.10.1989 zur Verabschiedung eines neuen Strafgesetzbuches, einer Strafprozeßordnung sowie eines Gerichtsverfassungsgesetzes und eines Gesetzes über die Staatsanwaltschaft gekommen war. Erstmals seit der Abschaffung aller Gesetze der Monarchie 1975 gelang es nach vierzehnjährigem gesetzlosen sozialistischem Interim, eine Neuverfassung des Justizwesens zu erreichen.

Der VE besteht aus einer Präambel und zehn Abschnitten mit insgesamt 73 Paragraphen. Die Abschnitte regeln folgende Verfassungskomplexe:

I. Allgemeine Grundlagen des Staates, hierzu gehören die (vorwiegend programmatische, von politischen Bekenntnissen geprägte) Präambel sowie die Abschnitte 1 bis 3

- Politisches System

- Wirtschafts- und Sozialstruktur

- Grundrechte und Grundpflichten der Bürger.

II. Verfassungsorgane (Abschnitte 4 bis 6)

- Oberste Volksversammlung

- Präsident der Republik

- Ministerrat. 
III. Die Abschnitte 7 bis 9 befassen sich mit

- lokaler Selbstverwaltung

- Justizwesen und

- Nationalen Emblemen.

IV. Abschnitt 10 enthält als Schlußbestimmung nur einen Paragraphen über die Verfassungsänderung.

Der VE dürfte mit seinen von sozialistischer Einheitsparteiherrschaft geprägten Vorstellungen einen eher anachronistisch anmutenden Text darstellen; in jeder Hinsicht ein Nachzügler unter den sozialistischen Verfassungen! Dennoch trägt er den Aspekten der gegenwärtigen laotischen Verfassungswirklichkeit Rechnung, mit der Betonung der Rolle der Volksvertretung eröffnet er einen parlamentarischen Ansatz und gibt Laos' Bürgern eine Reihe von Grundrechten, die über ein Jahrzehnt nach der sozialistischen Machtübernahme nicht nur de facto suspendiert waren, sondern teilweise so wenig eingehalten wurden, daß Laos sich lange Jahre vor den VN-Menschenrechtsgremien verantworten mußte.

1. Die Präambel und § 1 etablieren die ausschlaggebende Rolle der Laotischen Revolutionären Volkspartei (LRV), der Einheitspartei, die aus der ehemaligen Kommunistischen Partei Indochinas hervorgegangen ist. Laos ist dementsprechend eine Volksdemokratie $(\S \S 1,4,7)$, die auf dem Regime des Demokratischen Zentralismus ( $(6)$ beruht. Der laotische Staat ist ein unteilbarer pluriethnischer Einheitsstaat (\$2). Die Idee des Zentralstaates erfährt im VE eine beträchtliche Relativierung durch die Anerkennung der pluriethnischen Struktur des laotischen Volkes und durch die Grundsätze der Selbstverwaltung ( $\S 50 \mathrm{ff}$.), die der de-facto-Autonomie der Provinzen Rechnung trägt; legislativen und exekutiven Selbstverwaltungsgremien eignen beträchtliche Kompetenzen. Die Idee der pluriethnischen Zusammensetzung der laotischen Bevölkerung ist zum Verfassungsprinzip von primordialem Rang erhoben worden: Mindestens ein Dutzendmal wird es im VE genannt (Präambel, $\S \S 2,3,8,9,14,15,43$ ). Die Gleichheit aller ethnischen Gruppen wird besonders betont ( $\$ 9)$.

Eine textlich nicht derart ausführliche, aber der Verfassungswirklichkeit gerechtwerdende Rechtsposition wird der (buddhistischen) Religionsausübung und der Religionsfreiheit eingeräumt ( $\S 10,26)$. Eine verfassungsrechtlich anerkannte Stellung nehmen auch die gesellschaftlichen Massenorganisationen (Nationale Front, Gewerkschaftsbund, Revolutionäre Jugend Organisation und Laotischer Frauenverband) ein. Als grundlegende Verfassungsprinzipien sind die Gesetzmäßigkeit der Verwaltung (§ 11), die Nationale Verteidigung $(\S 12)$ und eine friedliche, blockfreie Außenpolitik anerkannt (§ 13). Maßgebend für die Außenpolitik sind die fünf Prinzipien der friedlichen Koexistenz, Respektierung von Unabhängigkeit, Souveränität und territorialer Integrität, der Nichteinmischung und der internationalen Zusammenarbeit auf der Grundlage 
von Gleichheit und gegenseitigen Vorteils. Ausdrücklich wird daneben die "aktive Unterstützung des Kampfes der Völker für Frieden, nationale Unabhängigkeit, Demokratie und sozialen Fortschritt" als Richtlinie der Außenpolitik genannt. Unter den 73 Staaten, mit denen Laos diplomatische Beziehungen unterhält, überwiegen deutlich solche des einstigen sozialistischen Lagers und die "fortschrittlichen" Länder der Dritten Welt.

2. Im zweiten Abschnitt des VE, übertitelt "Wirtschafts- und Sozialstruktur", stehen sich überkommene sozialistische und marktwirtschaftliche Prinzipien gegenüber: Artikel 17 des Entwurfs versucht, staatliche Planifikation, die als Wirtschaftsprinzip die Verfassungsorgane bindet (vgl. § 32 Ziffer 4, §46 Ziffer 4, § 52 Ziffer 5, § 55 Ziffer 6), mit der Marktwirtschaft zu verbinden. Marktwirtschaft wird im VE primär als höhere Stufe gegenüber der in Laos noch weitgehend herrschenden Naturalwirtschaft verstanden (§14). Als Entwicklungsmaßnahme bekennt sich der VE ausdrücklich zur Förderung der Wirtschaftsbeziehungen mit dem Ausland ( $\$ 14$ Abs. III), zum Investitionsschutz ausländischen Anlagevermögens (Art. 15) und zum Schutz der Rechte des Ausländers in Laos allgemein ( $30 \mathrm{Abs}$. I).

Neben das Entwicklungspostulat ( $(14)$ stellt der VE eine Garantie des Privateigentums $(\S \S 15,16)$, das als gleichberechtigte Eigentumsform neben Staats- und Kollektiveigentum sowie den Mischformen staatlichen und privaten Eigentums genannt wird. $\S 14$ sanktioniert so die im Investitionsgesetz vom Juli 1988 genannten Eigentumsformen und bekräftigt die darin enthaltenen Eigentumsgarantien, indem er sie verfassungsrechtlich absichert. $\mathrm{Ob}$ die verfassungsrechtliche Verankerung des Schutzes von ausländischen Investitionen den bisher schleppend verlaufenden Investitionsprozeß befördem wird, bleibt abzuwarten.

Die Reihe gegensätzlich anmutender wirtschaftspolitischer Zielsetzungen im VE läßt sich fortsetzen: hier Schutz von Eigentum und Erbrecht ( $\$ 16)$, Anerkennung des freien Wettbewerbs ( $\$ 15$ Abs. III), dort staatlicher Eingriff, zentralistische Reglementierung. Der Staat realisiert den Wirtschaftsmechanismus in Anwendung des Prinzips des "demokratischen Zentralismus" (§ 17), dem Staat obliegen Umweltschutz (§ 18), das gesamte Erziehungs- und Gesundheitswesen $(\$ \S 19,20)$. An Grund und Boden, der grundsätzlich dem Staat gehört, hat der Einzelne nur ein -allerdings vererbliches Nutzungsrecht. Im öffentlichen Gesundheitswesen besteht ein verfassungsrechtliches Privileg für die Kämpfer der Revolution von 1975, ihrer Familien und Hinterbliebenen.

Im Erziehungswesen dominiert die Tyrannei der Werte: Der Staat entwickelt Kultur und nationale Traditionen in Übereinstimmung mit der "fortschrittlichen Kultur der Welt", er bekämpft "alle negativen Phänomene im ideologischen und kulturellen 
Bereich" (§ 19 Abs. I). Die staatliche Erziehung bildet den "Neuen Menschen im Einklang mit dem Volk" (§ 19 Abs. II).

3. Der Grundrechtskatalog ( $\S 21-31)$ enthält klassische individuelle Freiheitsrechte und Grundpflichten, jedoch ohne Einklagbarkeit: Gleichheit vor dem Gesetz (§21), Gleichberechtigung der Geschlechter ( $\$ 22$ ), allgemeines aktives und passives Wahlrecht (§ 21), Freiheit der Berufsausübung (§23). Die sozialen Rechte der Arbeitnehmer enthalten kein Streikrecht, sie unterfallen wie die Freiheit der Berufsausübung dem Gesetzesvorbehalt. Der Gesetzesvorbehalt gilt auch für das Petitions- und Beschwerderecht (§24), das Recht auf körperliche Unversehrtheit und die Unverletzlichkeit der Wohnung (§ 25), für Glaubensfreiheit, Redefreiheit, Pressefreiheit, Versammlungs- und Vereinigungsfreiheit sowie Demonstrationsfreiheit (§ 26). Ohne Gesetzesvorbehalt gilt die Freiheit von Kunst und Wissenschaft.

§ 27 zieht erstmals aus der massiven Flucht von einem Zehntel der Bevölkerung nach dem Sturz der Monarchie und der Einrichtung der Volksrepublik Laos Konsequenzen aus dem Massenexodus, indem er die legitimen Rechte und Interessen der Auslandslaoten in allgemeiner Form anerkennt und unter staatlichen Schutz stellt. Offensichtlich soll mit dieser Bestimmung ein Anreiz zur Rückkehr verfassungsrechtlichen Rang erfahren. Bisher sind rund 20.000 Personen, darunter 5.000 im Rahmen des freiwilligen Wiederansiedlungsprogrammes der Hohen Flüchtlingskommission zurückgekehrt. $\mathrm{Ob}$ es gemäß $§ 27$ VE zur Ausstellung von Personalpapieren, Urkundenbeschaffung (die für Flüchtlinge bisher nicht erhältlich sind) und Eigentumsrestitution (zumindest bei Rückkehr) von Auslandslaoten kommt, bleibt abzuwarten.

$\mathrm{Zu}$ den statuierten Grundpflichten der Staatsbürger gehören die allgemeine Wehrpflicht (§ 29), die Verfassungstreue, Steuerpflicht, Bewahrung von Ruhe und Ordnung und Beachtung der Regeln des gesellschaftlichen Lebens sowie die Pflicht zur Arbeitsdisziplin (§ 28).

Artikel 30 beinhaltet das Ausländerstatut. Ausländer und Staatenlose in Laos genießen Eigentums- und Rechtsschutz, sie haben Zugang zu den Gerichten und Behörden mit ihren Anliegen und müssen Verfassung und Gesetze achten. Laos gewährt ein eingeschränktes Asylrecht für Verfolgte, die wegen ihres Einsatzes für Freiheit, Gerechtigkeit, Frieden und wissenschaftliche Bestrebungen Asyl begehren (Art. 30 Abs. II). Bisher gibt es keine Asylanten im Lande. 


\section{Verfassungsorgane}

1. Unter den Verfassungsorganen nimmt das Parlament, die Oberste Volksversammlung (OVV), als höchste Repräsentanz des Volkes, ausgestattet mit der legislativen Gewalt und zugleich als Oberste Kontrollinstanz des staatlichen Lebens, eine herausgehobene Stellung ein (\$31). Allein die OVV ist mit Zweidrittel-Mehrheit ihrer Mitglieder zur Verfassungsänderung befugt ( $\$ 32$ Ziffer 1,73 ). Der enumerative Kompetenzkatalog des $\$ 32$ des VE enthält in Ziffer 13 eine Generalklausel, wonach die OVV alle Rechte und Aufgaben wahmimmt, die sie als ihre Befugnisse selbst festsetzt, wobei man davon ausgehen muß, daß dies unbeschadet der verfassungsmäßigen Rechte der übrigen Verfassungsorgane gilt.

Neben der allgemeinen gesetzgebenden Gewalt ( $\$ 32$ Ziffer 2 ) besitzt die OVV insbesondere das Budgetrecht, das Recht der Feststellung der Entwicklungsplanung, die Kompetenz, Provinz- und Präfekturgrenzen zu ändem, die Steuergesetzgebung, die Amnestieregelung, das Recht, über Krieg und Frieden zu entscheiden, das Recht zur Ratifizierung völkerrechtlicher Verträge.

Als oberster Kontrollinstanz obliegt der OVV die Kontrolle über die verfassungsmäßige Anwendung der Gesetze ( $\$ 32$ Ziffer 3). Sie wählt und kann die eigenen Organe abberufen, wie den Parlamentspräsidenten, die Vizepräsidenten sowie die Mitglieder des Ständigen Ausschusses des Parlaments, aber auch den Staatspräsidenten, den Ministerpräsidenten, den Präsidenten des Obersten Volksgerichts wie den Generalstaatsanwalt ( 32 Ziffer 5). Die OVV setzt ihre ständigen Kommissionen ein ( $\$ 32$ Ziffer 5, 36 Ziffer 2) und entscheidet auf Vorschlag des Ministerrats über Bildung und Auflösung von Ministerien und Staatskomitees. Es ist evident, daß das Parlament mit seiner Kompetenzfülle weit in den herkömmlichen Bereich der Exekutive übergreift. Im Gegensatz dazu steht, daß die OVV nur zweimal im Jahr durch den Ständigen Ausschuß zu ordentlichen Sitzungsperioden zusammengerufen wird ( 34 Abs. V). Bisher nahmen die ordentlichen Sitzungsperioden jeweils nicht einmal die Dauer einer Woche ein. Außerordentliche Sitzungen können auf Verlangen einer qualifizierten Mehrheit von zwei Dritteln der Mitglieder der OVV einberufen werden ( 34 Abs. III).

Das Quorum für die Beschlußfähigkeit der OVV beträgt mehr als die Hälfte ihrer Mitglieder. Úber ihre Zahl resp. die Zahl der Wahlkreise trifft der VE keine Bestimmung. Gesetze und Beschlüsse der OVV erlangen Rechtsgültigkeit mit einfacher Mehrheit der anwesenden Mitglieder ( $\$ 37$ Abs. I). Eine qualifizierte Mehrheit verlang lediglich die bereits zuvor genannte Bestimmung des $\S 73$ über Verfassungsänderungen. Das Recht zur Gesetzesinitiative ( $\$ 36$ ) besitzen der Ständige Ausschuß, der Ministerrat, der Oberste Gerichtshof und der Generalstaatsanwalt sowie die gesellschaftlichen Massenorganisationen ( $\$ 8)$. 
Die Abgeordneten werden für eine Legislaturperiode von fünf Jahren gewählt ( $\$ 33$ Abs. I), ihr Mandat kann sich in Kriegs- und Krisenzeiten verlängem, endet jedoch spätestens 6 Monate nach Wiedereintreten normaler Verhältnisse ( 33 Abs. II). Sie genießen während der Dauer ihres Mandats Immunität und Indemnität (§ 42), können aber jederzeit gemäß $\$ 5$ Abs. II VE auf Vorschlag des Wählers wegen mangelhafter Mandatsausübung (d.h. "wenn sie wegen schlechter Konduite nicht mehr das Vertrauen des Volkes verdienen") ihr Mandat verlieren. Das Verfahren ist im VE nicht im einzelnen geregelt, ergibt sich aber aus $\S 32$ Ziffer 5,13 in Verbindung mit $\S 38$ Ziffer 5 und 40 Abs. II VE.

Die parlamentarische Verantwortlichkeit der Regienung ergibt sich aus $\S 41$ in Verbindung mit $\S \S 45$ Abs. II, 31, 32 Ziffer 5, § 38 Ziffer 6. Die Verantwortlichkeit der Justiz ist analog geregelt ( $\$ 57$ Abs. II, 58 Abs. II, 65 Abs. I und II).

2. Der VE hat das Zweikammersystem der königlichen Verfassung von 1947 nicht übernommen. Aber wenn die alte Verfassung den Königlichen Rat als Oberhaus der Nationalversammlung kannte, dessen 12 Mitglieder zur Hälfte gewählt, zur Hälfte vom König emannt wurden, so hat der VE von 1990 mit der Instituierung des Ständigen Ausschusses ein exekutives Instrument der OVV geschaffen, das eine Reihe von z.T. sehr weitgehenden originären Kompetenzen vereint ( $\$ 38$ Ziffer 1-18), darunter die Emennung und Abberufung laotischer Diplomaten im Ausland, Gewährung oder Entzug der laotischen Staatsangehörigkeit, Festsetzung des Ranges der Offiziere ab dem Obersten, Verleihung von Orden und Ehrenzeichen. Im eigenen organisatorischen Bereich kommt dem Ständigen Ausschuß das Recht zur Einberufung der OVV, die Festsetzung ihres Wahltermins sowie der sitzungsfreien Perioden des Parlaments zu, in der legislativen Domäne besitzt der Ausschuß das Verordnungsrecht, das Recht zur authentischen Verfassungsinterpretation, das Recht zur Änderung normativer Akte des Ministerrats, d.h. diese aufzuheben, aufzuschieben oder sie inhaltlich zu ändern. Im Bereich der Auswärtigen Gewalt das Recht zur Ratifizienung völkerrechtlicher Verträge mit dem Ausland oder aber diese abzulehnen unter dem Vorbehalt, sie der OVV zur Prüfung und Entscheidung vorzulegen. Im übrigen kann der Ständige Ausschuß alle ihm sonst von der OVV delegierten Kompetenzen ausüben.

Die sehr weitgehenden Befugnisse des Ausschusses erklären sich aus der bisherigen Verfassungspraxis extrem kurzer Sitzungsperioden der parlamentarischen Vollversammlung.

Die Stellung der OVV erscheint in Relation zur Position der übrigen Verfassungsorgane besonders stark. Im Vergleich mit der königlichen Verfassung von 1947 mutet sie eher unbalanciert, ideologisch zugespitzt und somit überpointiert an. So fehlen alle 
analogen Regelungen über ein Gegengewicht der Exekutive wie das einstige königliche Notverordnungsrecht und die Ausübung des Notstands.

Auch das Recht der vorzeitigen Parlamentsauflösung der Monarchen (auf Vorschlag des Ministerrats) ist entfallen und nicht auf das präsidiale Staatsoberhaupt übergegangen. Die Auflösungsdrohung schränkte in der Monarchie parlamentarische Kontrolle und Verantwortlichkeit der Regierung gegenüber dem Parlament in der Verfassungswirklichkeit erheblich ein.

3. Dem Staatsoberhaupt, dem Präsidenten der Republik, widmet der VE ganze zwei Paragraphen, seine Rolle ist auf rein repräsentative Funktionen beschränkt: Er wird nicht vom Volk, sondern von der OVV gewählt ( $\S 32$ Ziffer 5, 43) und repräsentiert als Staatschef das pluriethnische laotische Volk nach innen und außen.

Ganz offensichtlich lag dem Verfassungsgeber daran, jegliche Assoziation mit der verfassungsmäßigen Rolle des einstigen Monarchen auszuschließen. Der laotische König spielte nicht nur eine bedeutsame symbolische Rolle in Staat und Volksbewußtsein, er stellte darüber hinaus einen Faktor von verfassungsrechtlichem und politischem Gewicht dar: Er war Oberbefehlshaber der Armee, Gesetzgeber in Zeiten des Notstandes, konnte direkte exekutive Gewalt ausüben, den Sitzungen des Ministerrats präsidieren und besa $\beta$ das Begnadigungsrecht sowie das bedeutsame Recht der Parlamentsauflösung. $§ 44$ des VE beläßt dem Staatsoberhaupt nur noch einen Schatten der einstigen monarchischen Gewalt. Entsprechend den Entscheidungen des OVV promulgiert der Präsident Gesetze und Verordnungen, ratifiziert völkerrechtliche Verträge, ernennt die laotischen Missionschefs im Ausland und beruft sie ab, empfängt ausländische Missionschefs und promulgiert Amnestiegesetze, Mobilisierung und Kriegserklärung: Der Staatspräsident funktioniert als oberster Staatsnotar.

4. Die intendierte verfassungsmäßige Schwächung der Exekutive wird auch an der Stellung des Ministerrats deutlich. Als Kollektivorgan bildet der Ministerrat die Regierung des Staates und verkörpert sohin die oberste exekutive und administrative Gewalt ( $\$ 45$ Abs. I). Der Ministerrat besteht aus dem direkt von der OVV gewählten Ministerpräsidenten ( $\S 32$ Ziffer 5, 45 Abs. II), den Vizepräsidenten, den Ministern sowie den Präsidenten des Staatskomitees, denen § 45 Abs. II Kabinettsrang zuerkennt. Der Ministerpräsident bestimmt und leitet die Arbeit des Ministerrats, er bestimmt die Richtlinien der Politik. Seine Entscheidungen ergehen in Form der Dekrete und Verwaltungsanweisungen ( $\$ 48)$. Kollektiventscheidungen des Ministerrats in Form der vorgenannten Normen besitzen vorbehaltlich der Regelung des $\S 38$ Ziffer 7 exekutive Kraft für das genannte Staatsgebiet (§ 47). Der Ministerpräsident kann einen Vizepräsidenten für den Fall seiner Abwesenheit (und wohl auch bei Verhinderung) mit seiner Vertretung betrauen ( $\$ 48$ Abs. III). 
Obwohl der VE das Verfahren der Ministerberufung und Entlassung nicht ausdrücklich regelt, ist - argumentum e contrario - davon auszugehen, daß die Minister vom Ministerpräsidenten in eigener Verantwortlichkeit bestellt werden, lediglich der Ministerpräsident wird von der OVV direkt gewählt (s. oben). In der Praxis dürften sich angesichts der verfassungsrechtlich festgeschriebenen Einparteienherrschaft der Laotischen Revolutionären Volkspartei ( $\$ 1$ VE) derartige Entscheidungen im Zentralkomitee der Partei abspielen, ehe sie sich verfassungsrechtlich umsetzen. Zwar kann die OVV gemäß $\S 32$ Ziffer 7 neue Ministerien und Staatskomitees einrichten oder auflösen, jedoch nur auf Vorschlag des Ministerrats.

In der Tat zeigt die Regierung Kaysone Phomvihane seit der ersten Kabinettsbildung vom 4.12.1975, die das 9. Kabinett Souvanna Phouma und damit das letzte Kabinett der Monarchie ablöste, eine beachtenswerte personelle Kontinuität: Ministerpräsident, Verteidigungsminister und Justizminister haben seither nicht gewechselt. Gegenwärtig besteht die Regierung aus 14 Ministern.

Die - eher verborgene - Stärke der Regierung zeigt sich in der Regelung ihrer parlamentarischen Verantwortlichkeit. Der Ministerrat ist der OVV verantwortlich, der er in der Sitzungsperiode Rechenschaft ablegt, in der sitzungsfreien Zeit tritt an die Stelle des Gesamtparlaments der Ständige Ausschuß ( $\$ 45$ Abs. IV). Grundsätzlich entspricht die Amtsdauer der Regierung dem Mandat der OVV, d.h. sie amtiert für fünf Jahre ( $\$ 45$ Abs. V). Der VE kennt dementsprechend kein Verfahren der Vertrauensfrage (und daher auch nicht die Institution des care taker Govemment). Dennoch eröffnet die Regelung der §§ 31, 32 Ziffer 5, 37 Abs. I, 41, 45 Abs. IV die Möglichkeit des Sturzes des Ministerpräsidenten und damit die Ablösung der Regierung mit einfacher Mehrheit. Die bisherige "Verfassungswirklichkeit" spricht hingegen eher dafür, daß die Vorentscheidung, wenn überhaupt, im ZK der Einheitspartei fällt.

Der Kompetenzkatalog des $\S 46$ VE gibt der Regierung bei generalklauselhafter Umschreibung im einzelnen klar definierte und limitierte Aufgabenbereiche.

Im Verhältnis zur OVV besitzt der Ministerrat ein Initiativrecht für Gesetze und Verordnungen, er legt ihr den Budgetentwurf und den volkswirtschaftlichen Entwicklungsplan vor. Der Ministerrat kann die Anwendung der von provinzial- und präfekturalen Volksvertretungen beschlossenen Normen einstweilig aussetzen und der OVV zur endgültigen Beschlußfassung vorlegen.

Im klassischen Exekutivbereich besitzt der Ministerrat Organisations- und Kontrollgewalt gegenüber den Ministerien und den nachgeordneten Behörden, insbesondere in Hinsicht auf Planerfüllung und Einhaltung des Budgets, allgemeine Polizeigewalt zum Schutz der Staatsbürger sowie zur Aufrechterhaltung von Ruhe und Ordnung. Er hat 
das Kasşationsrecht in bezug auf Entscheidungen der nachgeordneten Behörden. Eine Verfassungs- oder Verwaltungsgerichtsbarkeit besteht nicht. Besonders hervorgehoben werden die Zuständigkeit der Regierung für Armee und Landesverteidigung, Abschluß völkerrechtlicher Verträge sowie das Recht, die Distriktgrenzen zu bestimmen.

Angesichts des Fehlens einer allgemeinen Ersatzzuständigkeit ist von der Ausschließlichkeit der zugeordneten Befugnisse auszugehen. Das Enumerativprinzip für die Domäne der Exekutive wirkt eher wie eine willkürliche Einzelfallregelung denn als ausgewogene Kompetenzverteilung zwischen Exekutive und Legislative, zwischen Zentral- und Provinzverwaltung.

\section{Territoriale Gliederung, Selbstverwaltung und Justiz}

Laos ist ein dezentralisierter Einheitsstaat, der in Provinzen gegliedert ist ( $\S 2,50 \mathrm{VE}$ ). Das einheitsstaatliche Element betont auch die Präambel (Abs. V) bei aller Anerkennung der pluriethnischen Verschiedenheiten. Die gegenwärtig 17 Provinzen (khoueng) stellen die großen gewachsenen Verwaltungseinheiten dar, sie gliedern sich in Distrikte (tasseng) und diese wiederum in Gemeinden (ban). Der VE nennt noch als weitere Verwaltungsebene unterhalb der Provinz die Präfektur; jedoch lediglich innerhalb der Provinz Vientiane stellt die Präfektur gleichen Namens mit der. Hauptstadt eine eigene - von der Provinz getrennte Verwaltungseinheit dar, andere Provinzen kennen keine gesonderten Präfekturen.

Jede Provinz, Präfektur oder Distrikt besitzen jeweils Volksvertretung und Verwaltungskomitee auf entsprechender Ebene. Von der traditionellen französischen Verwaltungspraxis her stellt sich Laos konzeptionell als straff gelenkter Zentralstaat dar. In der Praxis war es weder zur Zeit der Monarchie noch in der volksdemokratischen Republik jemals besonders gut um die Kontrolle der Zentralregierung hinsichtlich der Provinzen bestellt (selbst wenn man die historische Teilung in die einstigen drei Königreiche von Luang Prabang, Vientiane und Champassak und die durch die 1. Genfer Indochina-Konferenz 1954 de facto bewirkte Autonomie der Pathet Lao Provinzen Houa Phanh und phongsaly außer acht läßt).

1. Die Provinzial- und Distriktvolksvertretungen repräsentieren die lokalen Staatsorgane (Art. 51 Abs. I). Sie entscheiden über Fragen der wirtschaftlichen und kulturellen Entwicklung, sind für die Aufrechterhaltung von Ruhe und Ordnung in ihren jeweiligen Bereichen zuständig ebenso wie für den Schutz der Bevölkerung. Sie führen die ihnen von höheren Instanzen übertragenen Aufgaben durch (§ 51 Abs. II).

Sie werden für die Dauer von fünf Jahren gewählt, lediglich das Mandat der Distriktabgeordneten beläuft sich auf zweieinhalb Jahre. Eine Abwahl oder Absetzung durch die OVV ist nicht vorgesehen. Jedoch kann die OVV gemäß $\S 37$ Ziffer 8 die bestehen- 
den Provinzgrenzen ändern resp. neue Provinzen schaffen. Die lokalen Volksversammlungen unterliegen der Kontrolle durch den Ständigen Ausschuß des Parlaments ( 38 Ziffer 6 und 7), der ihre normativen Akte, sofern sie gegen die Verfassung oder die bestehenden Gesetze verstoßen, ändern resp. aufheben kann.

Nachgeordneten Volksvertretungen in Provinzen und Distrikten sind durch $\S 52$ folgende Kompetenzen zugewiesen:

- Anwendung von Verfassung und Gesetz auf ihrer jeweiligen lokalen Ebene,

- Bestimmung und Amtsenthebung der Mitglieder des Verwaltungskomitees sowie der Richter am Volksgericht auf Provinz- und Distriktebene,

- Aufsicht über den Geschäftsgang in Verwaltung und Justiz sowie der rangmäßig untergeordneten Volksversammlungen,

- Einrichtung von Anstalten und Dienstbetrieben der lokalen Verwaltung auf Vorschlag des Präsidenten des Verwaltungskomitees,

- Entscheidung über lokale Budgets und Entwicklungspläne und entsprechende Durchführungskontrolle,

- Änderung oder Kassation von nicht gesetzmäßigen Entscheidungen des Verwaltungskomitees oder einer rangniederen Volksversammlung,

- Schutz legitimer Interessen sowie Gewährleistung von Rechten und Pflichten der Staatsbürger,

- Beschwerdeinstanz im Rahmen der Gesetze,

- Wahl der jeweiligen ständigen Ausschüsse der lokalen Volksversammlungen.

2. Als Selbstverwaltungsorgan und staatliche Behörde zugleich ist das Verwaltungskomitee in den Provinzen und Distrikten an die Stelle des königlichen Gouvemeurs der Monarchie getreten. Dem Gouverneur als Organ der staatlichen Zentralverwaltung war Verfassungs- und Verwaltungsvollzug übertragen, er handelte auf Weisung des Innenministeriums. Der Gouverneur repräsentierte die Zentralregierung in seiner Provinz, er war Vorgesetzter für die Distrikt- und Gemeindeverwaltung, ohne Besteuerungs- und Ausgabenrecht war seine Macht auch in der Vergangenheit mehr nominell. Seit 1971 standen dem Gouverneur zwei Selbstverwaltungsorgane zur Seite: der 1949 geschaffene Provinzkonsultationsrat und der 1961 geschaffene Provinzialverwaltungsrat; diese Institutionen entsprachen in embryonaler Form Provinzial-Landtag und Kabinett.

Der VE, in seiner Tendenz durchweg legislativlastig, hat - wie zuvor gesehen - die Position der Provinzial-Landtage in der Form der lokalen Volksversammlungen entschieden aufgewertet, wohingegen an die Stelle von Gouverneur und Kabinett mit dem Verwaltungskomitee ein relativ machtloses reines Ausführungsorgan getreten ist.

Der Präsident des Verwaltungskomitees ist in seiner Stellung als Haupt einer Kollegialbehörde in keiner Weise dem königlichen Gouverneur gleichzusetzen, eigene 
Kompetenzen erkennt ihm die Verfassung (außer in $\$ 52$ Ziffer 4) nicht zu. Die Verwaltungskomitees haben eine dualistische Funktion: Sie sind Exekutivorgane der Selbstverwaltungskörperschaften wie staatliche Verwaltungsbehörden auf Provinzialund Distriktebene ( $\$ 54$ Abs. I). Ihre Amtszeit entspricht dem Mandat der entsprechenden Volksversammlung, sie unterstehen in ihrer Amtsführung der Kontrolle der jeweiligen lokalen Volksvertretung ( $\$ 54$ Abs. II, IV).

Der Kompetenzkatalog des $§ 57$ ist enumerativ und ausschließlich, eine Vermutung für subsidiäre Zuständigkeit ist nicht gegeben, der Wortlaut der Verfassung stützt die Annahme strikter Gesetzmäßigkeit der Verwaltung. Die Verwaltungskomitees besitzen im wesentlichen Aus- und Durchführungsbefugnisse aufgrund gesetzlicher Zuweisung. Auffällig sind die konkurrierenden Kompetenzen im Vergleich mit denen der lokalen Volksvertretungen (§ 52 Abs. VI zu § 55 Ziffer 7 und $8, \S 52$ Ziffer 8 zu § 55 Ziffer 9).

Der VE nimmt von der Gemeindeverfassung nicht Kenntnis. Hier gilt weiterhin noch der Dorfälteste als Partner der übergeordneten Distriktverwaltung. Die Gemeinde als unterste Verwaltungseinheit verbleibt angesichts des Schweigens des Verfassungsgesetzgebers im verfassungsfreien herkömmlichen Raum. Mit den Gemeindewahlen vom 26.6.1988, denen am 20.11.1988 Distrikt- und Provinzialwahlen folgten (vgl. Wahlgesetz vom 19.4.1988 und Ausführungs-VO vom 21.4.1988), setzte 13 Jahre nach der Machtübernahme durch die Laotische Revolutionäre Volkspartei der Prozeß der Demokratisierung ein, der mit den Wahlen vom 26.3.1989 zur OVV seinen bisherigen Höhepunkt erreichte.

3. Das Justizwesen hat in Kapitel VIII VE seine Regelung gefunden. Als Justizorgan werden die Volksgerichte, bestehend aus dem Obersten Volksgerichtshof und den Volksgerichten auf Provinz- und Distriktebene sowie den Militärgerichten (\$56) und die Volks-Staatsanwaltschaften ( $\$ 64)$ genannt. Als Organ der Rechtspflege findet auch die Rechtsanwaltsvereinigung Erwähnung ( $\$ 61$ Abs. II), dessen Funktion primär in der Rolle des Strafverteidigers garantiert wird.

Nach dem VE präsentiert sich die Demokratische Volksrepublik Laos nicht gerade als Justizstaat, und die Erscheinungsform des Sozialistischen Rechtsstaats, die die künftige Verfassung bietet, läßt an Montesquieu's Ausspruch über den pouvoir judiciaire im Rahmen der Gewaltenteilung als "en quelque facon nulle" denken. In den allgemeinen Bestimmungen der Anfangskapitel des VE findet die Justiz kaum Erwähnung ( $\S 21,3$ Abs. I).

Die richterliche Unabhängigkeit statuiert $\S 59$, jedoch haftet ihr ein momentaner Charakter an, wenn es heißt, "im Augenblick der Prüfung des Falles müssen Richter und (Laien)Beisitzer unabhängig sein und nur das Gesetz anwenden". Die Gerichte 
entscheiden als Kollegialinstanzen. Richter und Laienbeisitzer sind bei der Urteilsfindung gleichberechtigt (§60). Die Verhandlungen sind unbeschadet gesetzlicher Ausnahmeregelung (z.B. bei der Militärjustiz) grundsätzlich öffentlich (§1 Abs. I). Angeklagten steht das Recht der Verteidigung durch Anwälte zu (§61 Abs. II). Das Recht zur anwaltschaftlichen Vertretung ist auch zur Wahrung "sonstiger Interessen", d.h. also auch im Zivilverfahren gewährleiset (§ 61 Abs. II S. 2). Die Repräsentanten gesellschaftlicher Organisationen haben das Recht zur Mitwirkung in Gerichtsverfahren nach Maßgabe der Vorschriften ( $\$ 62$ ). In Betracht kommen die in $\S 8$ genannten Organisationen, wie insbesondere die Laotische Nationale Aufbaufront, die Laotische Revolutionäre Jugendorganisation, der Laotische Gewerkschaftsverband und die Laotische Frauenorganisation. Die Rechte der gesellschaftlichen Organisationen auf Beteiligung am justizförmigen Verfahren sind bisher nicht fixiert.

Der Oberste Volksgerichtshof sichert die Einheit der Rechtsprechung und fungiert als Appellationsinstanz gegenüber Entscheidungen der Untergerichte, die Militärgerichte eingeschlossen ( $\$ 58$ Abs. II). Obergericht wie die Volksgerichte in Provinz und Distrikt sind gegenüber der OVV bzw. den jeweils korrespondierenden lokalen Volksvertretungen verantwortlich und rechenschaftspflichtig (§58 Abs. III i.V. mit $\S 38$ Ziffer 6, 41, 52 Ziffer 3).

Die hierin zu erblickende Einschränkung der richterlichen Unabhängigkeit findet eine weitere Verschärfung durch das bis auf die Stufe der Untergerichte verfassungsrechtlich durchgeführte Prinzip der Richterwahl auf Zeit. Berufsrichter wie Laienbeisitzer werden jeweils durch die entsprechenden parlamentarischen Gremien gewählt. Die Dauer ihrer Amtsperiode entspricht dem zeitlichen Mandat der Abgeordneten (§ 57). Für die Wahl der Präsidenten und Vizepräsidenten und Richter der Obergerichte ist die OVV bzw. ihr Ständiger Ausschuß zuständig ( $\S 32$ Ziffer 5, 38 Ziffer 8), bei den Untergerichten die jeweils entsprechenden lokalen Volksvertretungen ( $\$ 52$ Ziffer 2). Über daraus resultierende Probleme, wie sich die zeitliche Befristung des Richteramts zur Fortsetzung schwebender Verfahren oder zur Gewährleistung des gesetzlichen Richters verhält, schweigt sich der VE aus.

Hinsichtlich der Rechtskraft und Durchsetzung richterlicher Entscheidungen bestimmt $\S 63$, daß diese von allen gesellschaftlichen Kräften zu respektieren seien, von allen Organisationen der Partei (d.h. der Laotischen Revolutionären Volkspartei) wie von den Staatsorganen und den Staatsbürgem. Vollstreckungen sind von den Betroffenen anzuerkennen, entsprechende Leistungen zu erbringen.

Der Generalstaatsanwalt und die Volksstaatsanwaltschaften sind mit der Kontrolle gerechter und einheitlicher Anwendung der Gesetze gegenüber jedermann beauftragt, d.h. gleichermaßen gegenüber Ministerien, Staatskomitees, Anstalten, Unternehmen, 
staatlichen und sozialen Einrichtungen, lokalen Verwaltungskomitees, Staatsangestellten wie Einzelpersonen ( $\$ 64)$. Während der Ausübung ihrer verfassungsmäßigen Funktionen unterstehen Staatsanwaltschaften und Militäranwaltschaften nicht den entsprechenden Provinzial- und Distriktgremien, d.h. auch nicht den jeweiligen Volksvertretungen.

Wie die Gerichte unterfallen Generalstaatsanwalt und Staatsanwaltschaften parlamentarischer Kontrolle (vgl. §§ 38 Ziffer 6, 41, 65) und sind - auf Zeit - gewählt (§§ 32 Ziffer 5, 38 Ziffer 8, 52 Ziffer 2, 65 Ziffem 1 und 3).

Der Oberste Gerichtshof und der Generalstaatsanwalt besitzen ein - nach dem Prinzip der Gewaltenteilung systemwidriges - Recht zur Gesetzesinitiative gegenüber der OVV (§36 Ziffer 4).

4. Kapitel IX trifft Bestimmungen über Schrift, Sprache, Nationalemblem, Nationalflagge, Nationalhymne und Hauptstadt. Wie in der Monarchie sind Schrift und Sprache laotisch, die Hauptstadt Vientiane und Hymne das Xat Lao (Laotische Nation), die aus dem Jahre 1943 datiert. Andere Symbole haben gewechselt: Statt der Fahne mit dem dreiköpfigen Elefanten ist die Flagge nunmehr rot-blau-rot mit weißem Kreis auf blauem Feld. Das Nationalemblem zeigt Stern, Hammer und Sichel, Reisfeld, Bäume und Staudamm anstelle des einstigen dreiköpfigen Elefanten unter weißem Parasol. 
The article deals with the first three areas of the law mentioned above, analysing the adequacy of recent constitutional amendments in the light of the influence of resurgent Islam on Egypt's political system.

\section{A Constitution for Laos}

\section{By Helmut Arndt}

Laos, placed between Thailand and Viemam in the north of Indochina, is a landlocked state difficult of access with a territory almost as large as the Federal Republic of Germany but with a population of a mere four million. Since the abolition of the monarchy in 1975 the country has been without a constitution. A draft constitution recently worked out by the Supreme People's Assembly is to be put into effect at the end of this year and will mark a new departure in the changeful history of the former "kingdom of a million elephants". 